\title{
遮阴对绣球光合特性和叶绿素荧光参数的影响
}

\author{
蔡建国" 韦孟琪 章 毅 魏云龙 \\ 浙江农林大学风景园林与建筑学院, 浙江临安 311300
}

\begin{abstract}
摘 要 为了从光合作用机制及叶片吸收光能分配的角度解释绣球(Hydrangea macrophylla)对不同光环境的适应机制, 探讨 绣球对光环境变化的生理响应和适应性，该文以盆栽的绣球品种“无尽夏新娘”为材料，设置遮阴(遮光率为 $50 \% 、 75 \%$ )两种处 理, 并以全光照为对照, 经过 60 天的处理, 测定其光合-光响应曲线、气体交换参数、叶绿素荧光参数。结果表明: 遮阴 60 天 后, 绣球的暗呼吸速率、光补偿点和光饱和点均有所下降, 而表观量子效率 $(A Q Y)$ 上升, 说明绣球能够通过这些途径提高对弱 光的利用能力并降低呼吸消耗, 以维持植株正常生长, 从而表现出了极强的适应能力; 在 $50 \%$ 的遮阴处理下, 绣球叶片的净 光合速率、胞间 $\mathrm{CO}_{2}$ 浓度、蒸腾速率和水分利用效率均与其全光照和 $75 \%$ 遮阴处理下差异显著; 遮阴导致光系统II (PSII)最大 光化学效率 $\left(F_{\mathrm{v}} / F_{\mathrm{m}}\right)$ 增加, 3 种光照处理下呈显著差异, 全光照下 $F_{\mathrm{v}} / F_{\mathrm{m}}$ 低于 $50 \%$ 遮阴处理, 初始荧光水平高于 $50 \%$ 遮阴处理, 推 断此条件下的绣球叶片发生了光抑制; 而随着光照的减弱, 非光化学淬灭系数在降低, 说明遮阴降低了绣球叶片PSII天线色 素吸收光能以热的形式耗散的比例, 绣球叶片吸收的能量约 $70 \%$ 用于热耗散, 约 $20 \%$ 用于非光化学反应, 仅有 $4 \%$ 的能量用于 光化学反应, 说明绣球处于饱和光环境下时, 主要通过提高叶片吸收光能向热耗散等PSII调节性能量耗散途径的分配, 削弱 反应中心过量激发能的积累。
\end{abstract}

关键词 绣球; 遮阴; 光合作用; 叶绿素苂光; 光适应

引用格式: 蔡建国, 韦孟琪, 章毅, 魏云龙 (2017). 遮阴对绣球光合特性和叶绿素苂光参数的影响. 植物生态学报, 41, 570-576. doi: 10.17521/cjpe. 2016.0245

\section{Effects of shading on photosynthetic characteristics and chlorophyll fluorescence parameters in leaves of Hydrangea macrophylla}

\author{
CAI Jian-Guo*, WEI Meng-Qi, ZHANG Yi, and WEI Yun-Long \\ School of Landscape Architecture, Zhejiang Agriculture and Forestry University, Lin'an, Zhejiang 311300, China
}

\begin{abstract}
Aims The objectives were to investigate the effects of different light intensities on photosynthetic characteristics and chlorophyll fluorescence parameters, to clarify the physiological responses and photo-protective mechanisms of Hydrangea macrophylla to changes in light regimes in view of the distribution of energy absorbed and photosynthetic characteristics.

Methods Three light regimes including natural and shade (shading rate $50 \%$ and $75 \%$ of natural light) were applied to plants for 60 days. After the treatment, the gas-exchange, chlorophyll a fluorescence and photosynthesis-light curves were measured by a portable leaf gas exchange system (LI-6400).

Important findings The results showed that the weak light intensity treatment reduced dark respiration rate, light compensation point and light saturation point of plant, but increased apparent quantum yield, suggesting that plants had the physiological strategy to utilize the weakening light by reducing respiration. The net photosynthetic rate, intercellular $\mathrm{CO}_{2}$ concentration, transpiration rate and water use efficiency of plants grown below $50 \%$ of natural light showed significant difference compared with natural and shading rate $75 \%$ of natural light. There were significant difference between natural and shade treatments in the maximal quantum efficiency of PSII $\left(F_{\mathrm{v}} / F_{\mathrm{m}}\right)$, as indicated that it was significantly less at full light than that at $50 \%$ of natural light. Initial fluorescence intensity $\left(F_{\mathrm{o}}\right)$ of plants was higher at full light than that at $50 \%$ of natural light, suggesting that photoinhibition occurred in natural light. The non-photochemical quenching $(N Q P)$ decreased with the aggravation of shade stress, indicating that shading decreased the efficiency of photochemical reaction by reducing the fraction of incident light in photochemical energy utilization and decreased thermal dissipation through regulating energy distribution in photosystem II (PSII) in the leaves of Hydrangea macrophylla. In general, the $70 \%$ of incident light in
\end{abstract}

收稿日期Received: 2016-07-18 接受日期Accepted: 2017-01-03

*E-mail: yuanlinzhiwu@163.com 
photochemical energy utilization was distributed to thermal dissipation, $20 \%$ was distributed to non-regulated energy dissipation and $4 \%$ was distributed to effective photochemical reaction. In conclusion, responses of plants to increased irradiance are governed by strategy: to utilize a high fraction of incident light in photochemistry and regulate energy dissipation in PSII and weaken the accumulation of excess excitation energy in PSII to protect the photosynthetic apparatus in the leaves of $H$. macrophylla under saturated radiation.

Key words Hydrangea macrophylla; shading; photosynthesis; chlorophyll fluorescence; light adaptation

Citation: Cai JG, Wei MQ, Zhang Y, Wei YL (2017). Effects of shading on photosynthetic characteristics and chlorophyll fluorescence parameters in leaves of Hydrangea macrophylla. Chinese Journal of Plant Ecology, 41, 570-576. doi: 10.17521/cjpe.2016.0245

光是植物光合作用和生长发育的重要影响因 子, 光强和光质的变化直接影响着植物的生长发育, 植物对光强、光质的变化也会做出灵活的响应(许大 全, 2013)。植物在长期的进化过程中, 由于不同植 物接收的太阳辐射量存在着明显差异, 因此形成了 自身特有的需光特性(张聪颖等, 2011)。植物经过遮 阴后, 叶片形态、光合生理参数会发生变化, 表现为 耐阴植物通常拥有较薄的颜色较深的叶片、较低的 暗呼吸速率、较高的光合效率、较高的光系统 II (PSII)效率以及较低的PSI/PSII (Wu et al., 2013)。 $\mathrm{C}_{3}$ 植物在弱光下均通过减少分枝、增加株高, 向高空 发展以截获更多光能(Poorther \& Perez-Soba, 2000)。 弱光条件下叶绿素含量增多是为了捕获更多的光能, 而植物的色素变化对光照的响应是不一样的, 绝对 的阴生植物其色素含量和构成都是不随光照条件变 化而变化的, 阳生植物通过增加叶绿素含量来适应 弱光条件, 中间型植物则是通过既改变叶绿素含量 也改变叶绿素构成来适应光照变化(Murchie \& Horton, 1998)。当光照强度超过植物的光饱和点时, 植物会发生光抑制或光破坏, 在中等光(稍高于 2 倍 生长光强, 生长光强: $\left.300-350 \mu \mathrm{mol} \cdot \mathrm{m}^{-2} \cdot \mathrm{s}^{-1}\right)$ 下, 大 豆(Glycine max) 叶片的光抑制主要是由于PSII的可 逆失活或下调, 与捕光天线LHCII的可逆脱离有关; 在强光(4倍或7倍于生长光强)下, 会造成大豆叶片 光破坏, 主要是PSII的不可逆破坏, 表现为叶绿素膜 蛋白(DI)发生净损失(Cai \& Xu, 2002)。在没有其他环 境胁迫因素存在的自然条件下, 即使太阳光足够强, 人们也不大容易看到植物发生不可逆光破坏的情况, 之所以如此, 是因为植物在长期的进化过程中已经 形成一系列防御光破坏的机制, 例如, 捕光天线脱 离反应中心复合体和状态转换(Hong \& Xu, 1999), 加强对过剩光能的热耗散, 加强光合作用对光能的 利用, 叶片和叶绿体的避光运动(Kagawa et al., 2001; Kasahara et al., 2002), 加强光呼吸等耗能代
谢, 加强对活性氧的清除和DI蛋白的周转等 $(X u \&$ Sheng, 1999)。在正常情况下, 这些机制足以保护光 合机构免于过量光能可能引起的不可逆破坏。

叶绿素苂光与光合作用中各个反应过程紧密 相关, 逆境对光合作用各过程产生的影响都可通过 植物体内叶绿素苂光诱导动力学变化反映出来(崔 晓伟等, 2011), 同时叶绿素荧光参数更反映植物“内 在性”的特点, 而且它具有快速、灵敏、非破坏性测 量等优点, 因而被视为研究植物光合作用与环境关 系的内在探针(Nedbal et al., 2000), 把叶片的荧光 分析与气体交换测定技术结合起来, 不仅可以从生 理学水平上更迅速、全面地了解光合机构的实际运 转状况, 而且可以间接地从生物化学水平上了解电 子传递速率及其与不同代谢过程的关系。

绣球 (Hydrangea macrophylla) 又名八仙花、粉团 花, 是虎耳草科绣球属植物, 高1-4 m, 常于基部发 出多数放射枝而形成一圆形灌从, 叶纸质或近革质, 倒卵形或阔椭圆形，花期6-8月，属暖温带半耐寒性 落叶小灌木(黄淑美等, 1995)。开花时花团锦簇, 颜 色丰富艳丽。花色会随着土壤 $\mathrm{pH}$ 值的变化而变成红 色或蓝色。由于绣球观赏效果较好且耐阴, 既可种 植于庭院、公园、绿地, 又能作盆花栽培, 在园林绿 化、美化中应用广泛(殷丽青等, 2010)。目前对绣球 的研究已经涉及栽培管理、花色花期调控、快繁技 术、抗性等方面, 但针对绣球的耐阴性研究鲜有报 道。本文以绣球品种“无尽夏新娘”为试验材料, 采用 模拟自然光照环境的方法, 从光合特性及叶绿素菼 光参数来探究绣球对弱光的适应程度, 旨在为绣球 的栽培和园林应用提供理论依据。

\section{1 材料和方法}

\section{1 试验材料}

试验于2014-2015年在浙江农林大学试验大棚 内进行, 以生长健壮、长势一致的绣球植株为材料, 
为保证绣球基因型一致, 2014年9月选取生长整齐一 致的插穗, 扞插于河沙中至生根, 10 月选取 60 株长 势良好且一致的生根苗移栽于上口径 $15.3 \mathrm{~cm}$ 、下口 径 $12.3 \mathrm{~cm}$ 、高 $17 \mathrm{~cm}$ 的容器, 单株定植, 栽培基质一 致。容器苗置于连栋大棚内, 进行常规栽培管理。 生长至2015年7月选用黑色遮阳网进行遮阴处理: 分别为遮光率 $50 \%$ 、遮光率 $75 \%$ 以及不遮光。本试 验采用随机区组试验, 既: 将受试材料, 分别随机 栽培于 3 个实验小区, 共 3 个光照处理, 每处理小区 5 株, 株行距各为 $20 \mathrm{~cm}$, 重复 3 次, 共计 9 个小区, 肥 水管理一致。60天后进行光合特性和叶绿素苂光参 数的测定。

\section{2 试验方法}

\subsection{1 光响应曲线的测定}

测定时间为2015年10月上旬晴天条件下, 应用 LI-6400便携式光合作用测定仪(LI-COR, Lincoln, USA)进行光响应曲线测定。 $\mathrm{CO}_{2}$ 浓度设定为 400 $\mu \mathrm{mol} \cdot \mathrm{mol}^{-1}$, 由系统 $\mathrm{CO}_{2}$ 注入系统自动控制; 叶室温 度控制在 $(25 \pm 0.5){ }^{\circ} \mathrm{C}$; 叶室相对湿度控制在 $(40 \pm$ $5) \%$; 光强梯度分别为 $2000 、 1500 、 1200 、 1000$ 、 $800 、 600 、 400 、 200 、 100 、 50 、 20 、 0 \mu \mathrm{mol} \cdot \mathrm{m}^{-2} \cdot \mathrm{s}^{-1}$ 。 选择中部同向新生叶片作为测定叶。首先对叶片进 行 $15 \mathrm{~min}$ 光诱导, 光强设置为 $1000 \mu \mathrm{mol} \cdot \mathrm{m}^{-2} \cdot \mathrm{s}^{-1}$ 。然 后开启光响应自动测量程序, 每个不同光强值设定 数据采集时间 $3 \mathrm{~min}$ 后, 进行测量。

\subsection{2 叶片气体交换参数的测定}

测定时间同上，应用LI-6400便携式光合作用 测定仪进行气体交换参数测定。各参数设置与光响 应曲线一致。光强设为 $1000 \mu \mathrm{mol} \cdot \mathrm{m}^{-2} \cdot \mathrm{s}^{-1}$, 每个处理 选择绣球各 3 株, 每株分别选择 3 个中部同向新生叶 片作为测定叶, 测定参数包括单叶净光合速率 $\left(P_{\mathrm{n}}\right)$ 、 蒸腾速率 $\left(T_{\mathrm{r}}\right)$ 、气孔导度 $\left(G_{\mathrm{s}}\right)$ 、胞间 $\mathrm{CO}_{2}$ 浓度 $\left(C_{\mathrm{i}}\right)$ 、叶 片温度 $\left(T_{1}\right)$; 主要环境影响因子, 包括水汽压亏缺 $(V P D)$ 、大气 $\mathrm{CO}_{2}$ 浓度 $\left(C_{\mathrm{a}}\right)$ 、光合有效辐射 $(P A R)$ 、气 温 $\left(T_{\mathrm{a}}\right)$ 、相对湿度 $(R H)$ 等参数。水分利用效率 $(W U E)=$ $P_{\mathrm{n}} / T_{\mathrm{r}}$ 。

\subsection{3 叶绿素荧光参数的测定}

用LI-6400便携式光合作用测定仪测定初始苂 光 $\left(F_{\mathrm{o}}\right)$ 、最大苂光 $\left(F_{\mathrm{m}}\right), \operatorname{PSII}$ 最大光化学效率 $\left(F_{\mathrm{v}} / F_{\mathrm{m}}\right)$, 光下最大荧光 $\left(F_{\mathrm{m}}^{\prime}\right)$ 和光下最小荧光 $\left(F_{\mathrm{o}}^{\prime}\right)$ 。测定 $F_{\mathrm{v}} / F_{\mathrm{m}}$ 前叶片充分暗适应 $30 \mathrm{~min}$ 。选择中部同向新生叶片 作为测定叶。每个处理选择绣球各3株, 每株测定3
个叶片, 每叶片重复3次。计算吸收光能用于天线热 耗散 $(D)=\left(1-F_{\mathrm{v}}^{\prime} / F_{\mathrm{m}}^{\prime}\right) \times 100 \%$, 光化学耗散 $(P)=q_{\mathrm{P}} \times$ $F_{\mathrm{v}}^{\prime} / F_{\mathrm{m}}^{\prime} \times 100 \%$, PSII反应中心非光化学能量耗散 $(E)=$ $\left(1-q_{\mathrm{P}}\right) \times F_{\mathrm{v}}^{\prime} / F_{\mathrm{m}}^{\prime} \times 100 \%$ (Ehlert \& Hinchaok, 2008; 崔晓伟等, 2011), $q_{\mathrm{P}}$ 为光化学淬灭系数。

\section{3 数据分析}

根据刘建锋等(2011)的方法拟合光响应曲线, 模型表达式为:

$$
\begin{aligned}
P_{\mathrm{n}} & =\frac{1}{2 \theta}\left(A Q Y P A R+P_{\mathrm{n} \max }-\right. \\
& \left.\sqrt{A Q Y P A R+P_{\mathrm{n} \max }^{2}-4 A Q Y P_{\mathrm{n} \max } \theta P A R}\right)-R_{\mathrm{d}}
\end{aligned}
$$

式中, $\theta$ 为光响应曲线的曲角; $A Q Y$ 为表观量子效率 $\left(\mathrm{mmol} \mathrm{CO} 2 \cdot \mathrm{mol}^{-1}\right.$ photos); $P_{\mathrm{nmax}}$ 为最大净光合速率 $\left(\mu \mathrm{mol} \quad \mathrm{CO}_{2} \cdot \mathrm{m}^{-2} \cdot \mathrm{s}^{-1}\right) ; R_{\mathrm{d}}$ 为暗呼吸速率 $\left(\mu \mathrm{mol} \quad \mathrm{CO}_{2}\right.$. $\left.\mathrm{m}^{-2} \cdot \mathrm{s}^{-1}\right)$ 。光补偿点 $\left(L C P, \mu \mathrm{mol} \cdot \mathrm{m}^{-2} \cdot \mathrm{s}^{-1}\right)$ 和光饱和点 $\left(L S P, \mu \mathrm{mol} \cdot \mathrm{m}^{-2} \cdot \mathrm{s}^{-1}\right)$ 是利用低光条件下 $(P A R \leqslant$ $\left.200 \mu \mathrm{mol} \cdot \mathrm{m}^{-2} \cdot \mathrm{s}^{-1}\right)$ 的 $P_{\mathrm{n}}$ 与 $P A R$ 构建线性方程 $\left(P_{\mathrm{n}}=\right.$ $\left.m P A R+n, L C P=-n / m, L S P=\left(P_{\text {nmax }}-n\right) / m\right)$ 所得。

利用Microsoft Excel 2007整理原始数据。用 SPSS 22.0软件进行方差分析, 采用Duncan氏新复 极差测验法进行多重比较。用光合计算4.0.1.软件辅 助计算光响应曲线拟合值, 利用Origin 8.0作图。

\section{2 结果}

\section{1 遮阴对光合-光响应曲线的影响}

光响应曲线可反映植物 $P_{\mathrm{n}}$ 随 $P A R$ 增减的变化规 律(李金鹏和董然, 2011), 由图1可知, 3种光照处理

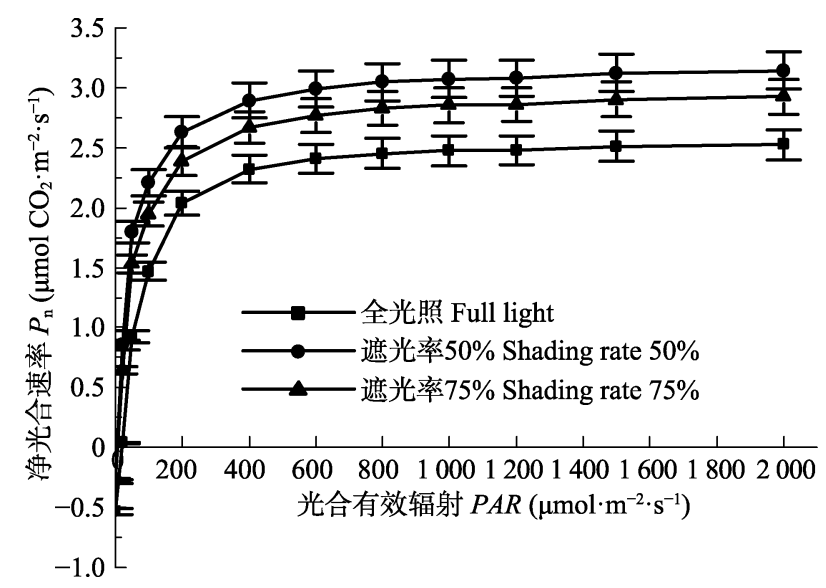

图1 不同遮阴处理下绣球叶片的光响应曲线(平均值土标准 偏差)。

Fig. 1 Light response curves of net photosynthetic rate $\left(P_{\mathrm{n}}\right)$ in Hydrangea macrophylla under different light treatments (mean $\pm S D)$. 
下的绣球光响应曲线变化规律相似, 当 $P A R$ 为 0 时, $P_{\mathrm{n}}$ 为负值, 随着 $P A R$ 的增强, $P_{\mathrm{n}}$ 逐渐增加, 由负值转 为正值, 当 $P A R$ 为 $400 \mu \mathrm{mol} \cdot \mathrm{m}^{-2} \cdot \mathrm{s}^{-1}$ 时, $P_{\mathrm{n}}$ 上升趋势减 缓并趋向平稳, 光响应曲线结果表明, 遮阴提高了 绣球的 $A Q Y$ 和 $P_{\mathrm{n} m a x}$, 并降低了 $L C P 、 L S P$ 和 $R_{\mathrm{d}}$ 。由表 1 可知, $50 \%$ 遮光率条件下的绣球叶 $R_{\mathrm{d}}$ 比全光照下降 低了 $31.4 \%, L C P$ 降低了 $57.4 \%, L S P$ 降低了 $9.5 \%$, 这 表明在遮阴环境下，绣球可通过降低 $L C P 、 L S P$ 来提 高对弱光的利用能力, 并通过降低呼吸消耗以维持 植株正常生长, 从而表现出了极强的适应能力。

\section{2 遮阴对叶片气体交换参数的影响}

由表 2 可知, $50 \%$ 遮光率处理下的绣球叶片的 $P_{\mathrm{n}}$ 和 $T_{\mathrm{r}}$ 分别高于全光照和 $75 \%$ 遮光率处理, 而 $C_{\mathrm{i}}$ 和 WUE低于全光照。 $50 \%$ 遮光率处理叶的 $P_{\mathrm{n}} 、 C_{\mathrm{i}} 、 T_{\mathrm{r}}$ 和 $W U E$ 与全光照叶差异显著, 其 $G_{\mathrm{s}}$ 与后者差异不显 著。说明 $P_{\mathrm{n}}$ 与 $T_{\mathrm{r}}$ 的变化一致, 遮阴增加了叶片的 $P_{\mathrm{n}}$, 较高的 $P_{\mathrm{n}}$ 伴随着较高的 $T_{\mathrm{r}}$ 。 $50 \%$ 遮光处理后, 叶片 $G_{\mathrm{s}}$ 没有发生变化, 但是 $W U E$ 降低了 $18.18 \%$, 说明遮 阴下的叶片需要消耗更多的水分以维持其高的净光

表1 不同遮光对绣球光响应特性的影响

Table 1 Effects of light treatments on photosynthesis-light response parameters of Hydrangea macrophylla

\begin{tabular}{|c|c|c|c|}
\hline $\begin{array}{l}\text { 参数 } \\
\text { Parameter }\end{array}$ & $\begin{array}{l}\text { 全光 } \\
\text { Full light }\end{array}$ & $\begin{array}{l}\text { 遮光率 } 50 \% \\
\text { Shading rate } \\
50 \%\end{array}$ & $\begin{array}{l}\text { 遮光率 } 75 \% \\
\text { Shading rate } \\
75 \%\end{array}$ \\
\hline $\begin{array}{l}\text { 表观量子效率 } \\
A Q Y\left(\mathrm{mmol} \mathrm{CO} \cdot \mathrm{mol}^{-1} \text { photos }\right)\end{array}$ & 0.03 & 0.04 & 0.03 \\
\hline $\begin{array}{l}\text { 最大净光合速率 } \\
P_{\mathrm{n} \max }\left(\mu \mathrm{molCO}{ }_{2} \cdot \mathrm{m}^{-2} \cdot \mathrm{s}^{-1}\right)\end{array}$ & 3.50 & 3.85 & 3.83 \\
\hline $\begin{array}{l}\text { 暗呼吸速率 } \\
R_{\mathrm{d}}\left(\mu \mathrm{molCO} \mathrm{C}_{2} \cdot \mathrm{m}^{-2} \cdot \mathrm{s}^{-1}\right)\end{array}$ & 0.53 & 0.36 & 0.41 \\
\hline $\begin{array}{l}\text { 光补偿点 } \\
L C P\left(\mu \mathrm{mol} \cdot \mathrm{m}^{-2} \cdot \mathrm{s}^{-1}\right)\end{array}$ & 15.75 & 6.71 & 8.00 \\
\hline $\begin{array}{l}\text { 光饱和点 } \\
\operatorname{LSP}\left(\mu \mathrm{mol} \cdot \mathrm{m}^{-2} \cdot \mathrm{s}^{-1} \mathrm{~s}^{-1}\right)\end{array}$ & 307.42 & 278.14 & 281.57 \\
\hline 相关系数 $r$ & 0.919 & 0.908 & 0.948 \\
\hline
\end{tabular}

$\overline{A Q Y \text {, apparent quantum yield; } P_{\mathrm{n}} \text {, net photosynthetic rate: } R_{\mathrm{d}} \text {, dark res- }}$ piratory rate; $L C P$, light compensation point; $L S P$, light saturation point.
合效率，而 $C_{\mathrm{i}}$ 降低说明叶片固定 $\mathrm{C}$ 的能力提高。 $75 \%$ 遮光率下的 $G_{\mathrm{s}}$ 与 $50 \%$ 遮光率和全光照下的差异显著, 下降了 $50 \%$, 说明过度遮阴影响绣球叶片气体交换 能力, 从而引起叶片的 $P_{\mathrm{n}}$ 下降。由此得出, 绣球在适 度遮阴后, 叶片会通过增加 $T_{\mathrm{r}}$ 和提高 $\mathrm{CO}_{2}$ 同化力来 提高 $P_{\mathrm{n}}$, 但过度遮阴会使叶片 $P_{\mathrm{n}}$ 下降, 原因是 $G_{\mathrm{s}}$ 降 低使得叶片气体交换能力下降。

\section{3 遮阴对叶绿素荧光参数的影响}

叶绿素荧光是光合作用的探针, 通过荧光参数 分析可以了解光合机构内部一系列重要的调节过程, $F_{\mathrm{v}} / F_{\mathrm{m}}$ 是PSII光能转化率的指标(缴丽莉等, 2007)。由 表3看出, 遮阴导致 $F_{\mathrm{v}} / F_{\mathrm{m}}$ 增加, 3种光照处理间差异 显著, 全光照下 $F_{\mathrm{v}} / F_{\mathrm{m}}$ 低于 $50 \%$ 遮光率处理, $F_{\mathrm{o}}$ 高于 $50 \%$ 遮光率处理, 根据张守仁(1999)的观点, $F_{0}$ 是判 断PSII反应中心运转情况的重要指标, $F_{\mathrm{o}}$ 上升表明 $\mathrm{PSII} 反$ 应中心受到破坏或失活, $F_{\mathrm{v}} / F_{\mathrm{m}}$ 下降是光抑制 的重要特征, 因此推测全光照下的绣球叶片发生了 光抑制。 $N P Q$ 的变化反映了非光化学耗散的能量, 其中包括了类囊体膜耗散热能, 是植物光合机构的 自我保护机制(何炎红等, 2006)。随着生长光照的减 弱, $N P Q$ 值在降低, 说明遮光处理降低了PSII天线 色素吸收光能以热的形式耗散的比例, 提高了PSII 吸收光能的利用效率。

\section{4 叶片吸收光能的分配}

植物叶片吸收的光能可分为 3 部分: 一是天线 热耗散的能量 $(D)$, 二是用于光化学反应的部分 $(P)$, 三是反应中心有非光化学反应耗散的能量 $(E)$ (Depuydt et al., 2009)。分析吸收光能的分配将有利于了 解植物叶片对吸收光能的分配策略(胡文海等, 2015)。由表4可知, $P$ 表现为: 50\%遮光率 > 全光照> $75 \%$ 遮光率。 $E$ 表现为: $75 \%$ 遮光率 $>50 \%$ 遮光率 > 全光照。 $D$ 表现为: 全光照和遮光率 $75 \%$ 的 $D$ 值高于 遮光率 $50 \%$, 由此看出, 遮光率 $50 \%$ 的绣球把吸收的

表2 不同遮光率处理对叶片气体交换参数影响(平均值土标准偏差)

Table 2 Effects of light treatments on the gas exchange parameters of Hydrangea macrophylla (mean $\pm S D$ )

\begin{tabular}{llllll}
\hline 处理 & \multicolumn{4}{c}{ 参数 Parameter } \\
\cline { 2 - 6 } & $P_{\mathrm{n}}\left(\mu \mathrm{mol} \mathrm{CO} \mathrm{CO}_{2} \cdot \mathrm{m}^{-2} \cdot \mathrm{s}^{-1}\right)$ & $C_{\mathrm{i}}\left(\mu \mathrm{mol} \cdot \mathrm{mol}^{-1}\right)$ & $G_{\mathrm{s}}\left(\mathrm{mol} \cdot \mathrm{m}^{-2} \cdot \mathrm{s}^{-1}\right)$ & $T_{\mathrm{r}}\left(\mathrm{mmol} \cdot \mathrm{m}^{-2} \cdot \mathrm{s}^{-1}\right)$ & $W U E\left(\mathrm{mmol} \mathrm{CO}_{2} \cdot \mathrm{mol}^{-1} \mathrm{H}_{2} \mathrm{O}\right)$ \\
\hline 全光 Full light & $1.64 \pm 0.34^{\mathrm{a}}$ & $326.55 \pm 21.45^{\mathrm{a}}$ & $0.04 \pm 0.004^{\mathrm{b}}$ & $0.92 \pm 0.11^{\mathrm{a}}$ & $1.82 \pm 0.53^{\mathrm{a}}$ \\
遮光率 $50 \%$ Shading rate $50 \%$ & $2.14 \pm 0.38^{\mathrm{b}}$ & $300.46 \pm 17.85^{\mathrm{b}}$ & $0.04 \pm 0.009^{\mathrm{b}}$ & $1.40 \pm 0.22^{\mathrm{b}}$ & $1.54 \pm 0.27^{\mathrm{b}}$ \\
遮光率 $75 \%$ Shading rate $75 \%$ & $1.73 \pm 0.28^{\mathrm{a}}$ & $293.99 \pm 18.33^{\mathrm{b}}$ & $0.02 \pm .009^{\mathrm{a}}$ & $0.86 \pm 0.26^{\mathrm{a}}$ & $2.11 \pm 0.47^{\mathrm{a}}$ \\
\hline
\end{tabular}

$C_{\mathrm{i}}$, 胞间 $\mathrm{CO}_{2}$ 浓度; $G_{\mathrm{s}}$, 气孔导度; $P_{\mathrm{n}}$, 净光合速率; $T_{\mathrm{r}}$, 蒸腾速率; $W U E$, 水分利用效率。同列不同字母表示处理间差异显著 $(p<0.05)$ 。

$C_{\mathrm{i}}$, intercellular $\mathrm{CO}_{2}$ content; $G_{\mathrm{s}}$, stomatal conductance; $P_{\mathrm{n}}$, net photosynthetic rate; $T_{\mathrm{r}}$, transpiration rate; $W U E$, water use efficiency. Different letters in same column indicate significant differences between treatments $(p<0.05)$. 
表3 不同遮阴处理对叶绿素苂光参数比较(平均值土标准偏差)

Table 3 Effects of light treatments on chlorophyll $a$ fluorescence parameters of Hydrangea macrophylla (mean $\pm S D$ )

\begin{tabular}{llll}
\hline 参数 Parameter & \multicolumn{2}{c}{ 处理 Treatment } \\
\cline { 2 - 4 } & 全光 Full light & 遮光率 $50 \%$ Shading rate $50 \%$ & 遮光率 $75 \%$ Shading rate $75 \%$ \\
\hline PS II 原初光能转化效率 $F_{\mathrm{v}} / F_{\mathrm{m}}$ & $0.63 \pm 0.03^{\mathrm{b}}$ & $0.67 \pm 0.01^{\mathrm{c}}$ & $0.60 \pm 0.04^{\mathrm{a}}$ \\
PS II 潜在活性 $F_{\mathrm{v}} / F_{\mathrm{o}}$ & $1.72 \pm 0.21^{\mathrm{b}}$ & $2.00 \pm 0.08^{\mathrm{c}}$ & $1.51 \pm 0.20^{\mathrm{a}}$ \\
初始苂光 $F_{\mathrm{o}}$ & $786.59 \pm 13.23^{\mathrm{b}}$ & $770.21 \pm 21.12^{\mathrm{a}}$ & $836.86 \pm 28.73^{\mathrm{b}}$ \\
PSII实际光量子产量 $F_{\mathrm{v}}^{\prime} / F_{\mathrm{m}}^{\prime}$ & $0.25 \pm 0.01^{\mathrm{a}}$ & $0.26 \pm .04^{\mathrm{a}}$ & $0.28 \pm 0.06^{\mathrm{a}}$ \\
非光化学淬灭系数 $N P Q$ & $2.39 \pm 0.16^{\mathrm{c}}$ & $1.95 \pm 0.09^{\mathrm{b}}$ & $1.41 \pm 0.34^{\mathrm{a}}$ \\
光化学淬灭系数 $q_{\mathrm{P}}$ & $0.17 \pm 0.04^{\mathrm{a}}$ & $0.18 \pm 0.02^{\mathrm{a}}$ & $0.15 \pm 0.03^{\mathrm{a}}$ \\
电子传递速率 $E T R$ & $19.43 \pm 3.88^{\mathrm{a}}$ & $20.31 \pm 5.53^{\mathrm{a}}$ & $18.57 \pm 4.50^{\mathrm{a}}$
\end{tabular}

ETR, electron transport rate; $F_{\mathrm{o}}$, initial fluorescence intensity; $F_{\mathrm{v}} / F_{\mathrm{m}}$, maximal quantum efficiency of PSII; $F_{\mathrm{v}} / F_{\mathrm{o}}$, potential efficiency of primary conversion of light energy of PSII; $F_{\mathrm{v}}^{\prime} / F_{\mathrm{m}}^{\prime}$, actual photochemical efficiency of PSII; $N P Q$, non-photochemical quenching; $q_{\mathrm{P}}$, photochemical quenching.

光能较多的分配于光化学反应部分, 而用于天线热 耗散的能量较少。说明在此处理下, 绣球叶片的光 化学反应能量利用率最大, 这与 $N Q P$ 变化一致。遮 光率 $75 \%$ 条件下，绣球由于光照不足，叶片吸收的 光能用于参与光化学反应耗散的份额降低。而较高 的 $E$ 值表明PSII光化学反应和保护性调节机制没有 发挥作用, 积累了大量的激发能, 这有可能会对 PSII反应中心产生破坏作用。全光照下，绣球叶片 $D$ 值较高, 说明叶片此时具有较高的可调控生理代谢, 能够将过剩的光能较多地分配给热耗散途径。而热 耗散比率的增加也反映了此时光能捕获效率降低, 推测植物有可能处于光抑制状态下(黄秋媡等, 2015)。因此, 绣球处于饱和光环境时, 绣球叶片吸 收的能量约 70\%用于热耗散, 约 $20 \%$ 用于非光化学 反应, 仅有 $4 \%$ 的能量用于光化学反应, 绣球的主要 光保护机制为PSII天线色素吸收光能通过热的形式 耗散, 以此削弱反应中心过量的激发能。

\section{3 讨论}

$L S P$ 和 $L C P$ 分别代表植物光合作用的光照强度 上限和下限, 同时也代表植物叶片对强光和弱光的 利用能力。耐阴植物的 $L C P$ 小于 $20 \mu \mathrm{mol} \cdot \mathrm{m}^{-2} \cdot \mathrm{s}^{-1}$, $L S P$ 为 50-1 $000 \mu \mathrm{mol} \cdot \mathrm{m}^{-2} \cdot \mathrm{s}^{-1}$ 或更低(Boardman,
$1977)$ 。绣球的 $L C P$ 为 $6.71-15.75 \mu \mathrm{mol} \cdot \mathrm{m}^{-2} \cdot \mathrm{s}^{-1}, L S P$ 为 $278.14-307.42 \mu \mathrm{mol} \cdot \mathrm{m}^{-2} \cdot \mathrm{s}^{-1}$ 。因此, 绣球属于耐阴 植物, 遮阴处理下的绣球 $R_{\mathrm{d}}$ 低于全光照下, 这说明 遮阴叶比全光照叶呼吸强度小, 绣球在遮阴环境下 能够通过降低 $L C P$ 和 $L S P$ 来增强对弱光的利用能力, 与王凯等(2009)的研究结果类似。 $A Q Y$ 反映了叶片 在弱光环境下的光合能力, 其值越大, 表明植物吸 收与转换光能的色素蛋白复合体越多, 植物利用弱 光的能力越强(Richardson \& Berlyn, 2002)。绣球在 弱光环境下的 $A Q Y$ 较全光照环境下高, 说明绣球对 弱光有较强的适应性。

正常情况下，没有遭受环境胁迫并经过充分暗 适应的植物叶片 $F_{\mathrm{v}} / F_{\mathrm{m}}$ 是比较恒定的, 一般为 0.80 0.85 (许大全, 2013), 但是本实验 3 种光照处理下绣 球的 $F_{\mathrm{v}} / F_{\mathrm{m}}$ 平均值仅为 0.63 , 显著低于正常值, $P_{\mathrm{n}}$ 值 也偏低, 分析原因可能是: 在试验中, 无法准确知 道绣球光诱导期的时间, 使得弱光下生长的植株较 长时间暴露在全光照下, 导致光合机构的过剩激发 能大幅增加, 最大光化学效率迅速降低, 因而出现 光抑制现象(李志真等, 2014)。由 $F_{\mathrm{v}}^{\prime} / F_{\mathrm{m}}^{\prime} 、 F_{\mathrm{v}} / F_{\mathrm{m}}$ 和 $F_{\mathrm{o}}$ 的降低, 可判断此时绣球的热耗散机制是依赖叶黄 素循环的能量耗散(许大全, 2013)。另一方面，由表3 和表 4 看出, 遮光率 $75 \%$ 的绣球 $E$ 值、 $F_{0}$ 最大, 说明较

表4 叶片吸收光能的分配

Table 4 The distribution of light energy absorbed in Hydrangea macrophylla under different light intensities

\begin{tabular}{|c|c|c|c|}
\hline \multirow{2}{*}{$\begin{array}{l}\text { 处理 } \\
\text { Treatment }\end{array}$} & \multicolumn{3}{|c|}{ 能量分配 Energy distribution } \\
\hline & $\begin{array}{c}\text { 天线热耗散 } \\
\text { Fraction of photons dissipated } \\
\text { in the antenna }(\%)\end{array}$ & $\begin{array}{l}\text { 光化学反应耗散 } \\
\text { Fraction of photons utilized } \\
\text { in PSII photochemistry (\%) }\end{array}$ & $\begin{array}{c}\text { 非光化学反应耗散 } \\
\text { Fraction of absorbed photons by PSII } \\
\text { neither used in photochemistry nor } \\
\text { dissipated in the PSII (\%) }\end{array}$ \\
\hline 全光 Full light & $74.55 \pm 5.32$ & $4.44 \pm 0.89$ & $21.01 \pm 1.12$ \\
\hline 遮阴 $50 \%$ Shading 50\% & $74.22 \pm 4.50$ & $4.65 \pm 1.27$ & $21.12 \pm 3.34$ \\
\hline 遮阴 $75 \%$ Shading 75\% & $74.55 \pm 5.32$ & $4.24 \pm 1.02$ & $23.64 \pm 6.13$ \\
\hline
\end{tabular}

www.plant-ecology.com 
其他两种光照处理, 受到的光抑制更加严重。一个 原因是 $E$ 值的增加会使单线态叶绿素 $\left({ }^{1} \mathrm{Chl}\right)$ 不能及 时返回三线态叶绿素 $\left({ }^{3} \mathrm{Chl}\right),{ }^{1} \mathrm{Chl}$ 的积累会将能量传 递给 $\mathrm{O}_{2}$, 形成活性极强的单线态氧 $\left({ }^{1} \mathrm{O}_{2}\right)$, 这会引起 光合机构的失活甚至破坏(Asada, 1999)。 $F_{\mathrm{o}}$ 的增加 说明植物叶片类囊体膜受到损害, PSII反应中心失 活(黄秋㜆等, 2015)。另一个原因是其阴生叶片的结 构和生理特征均缺少对强光的适应性, 所以遮阴下 生长的绣球叶片过剩激发能多, 光抑制程度也比直 接生长在全日照下的绣球要严重。这符合3种光照处 理下非光化学反应耗散变化趋势, 也与杨兴洪等 (2001)的研究结果一致。光合作用诱导期的长短差 别很大, 短的仅有几分钟, 长的可达 $1-2 \mathrm{~h}$ 。光诱导的 形成, 主要是来自光合碳同化酶的活化, 这些酶需 要光照作催化剂(许大全, 2003)。因此, 对如何控制 光照时间和光合同化酶的活性等因素使绣球达到合 适的光合诱导期有待继续探究。

综上所述, 绣球为耐半阴植物, 适度遮阴能够 提高其 $F_{\mathrm{v}} / F_{\mathrm{m}}$, 同时降低热耗散比例, 绣球在全光照 条件下很容易导致PSII反应中心失活而出现光抑制, 光合作用效率大幅度降低。在强光下绣球会启动光 保护机制, 是以热耗散途径为主的PSII调节性能量 耗散，遮阴 $75 \%$ 处理限制了光合碳同化酶的活性， 绣球光合性能并非最大, 因此绣球的最佳光照环境 为全光照的 $50 \%$, 在郁闭度为 0.5 左右时, 光合作用 捕获率最大, 有利于植物生长发育。

基金项目 国家自然科学基金(31272494)、浙江省 教改课题(KC14015和KC14032)、浙江省花卉团队项 目(2011R50034-02)和浙江省林学重中之重一级学 科研究生科研创新项目 (201531)。

致谢 感谢浙江农林大学风景园林与建筑学院的韦 孟琪、章毅在实验工作中给予的帮助。

\section{参考文献}

Asada K (1999). The water-water cycle in chloroplasts: Scavenging of active oxygens and dissipation of excess photons. Plant Biology, 50, 601-639.

Boardman NK (1977). Comparative photosynthesis of sun and shade plants. Plant Physiology, 28, 355-377.

Cai SQ, Xu DQ (2002). Light intensity-dependent reversible down-regulation and irreversible damage of PSII in soybean leaves. Plant Science, 163, 847-853.

Cui XW, Gao J, Zhang ZJ, Yue XH, Ma YJ (2011). Chlorophyll fluorescent characteristics of five dwarf bamboos.
Acta Agriculturae Universitatis Jiangxiensis, 33, 726730. (in Chinese with English abstract) [崔晓伟, 高健, 张 志坚, 岳祥华, 马艳军 (2011). 5种地被竹叶绿素荧光特 性研究. 江西农业大学学报, 33, 726-730.]

Depuydt S, Trenkamp S, Fernie AR, Elftieh S, Renou JP, Vuylsteke M, Holsters M, Vereecke D (2009). An integrated genomics approach to define niche establishment by Rhodococcus fascians. Plant Physiology, 149, 1366-1386.

Ehlert B, Hinchaok DK (2008). Chlomphyll fluorescence imaging accurately quantifies freezing damage and cold acclimation responses in Arabidaopsis leaves. Plant Methods, 4, 1-7.

He YH, Guo LS, Tian YL (2006). Chlorophyll fluorescence quenching characteristics of seven coniferous and broadleaved species in different light intensities. Scientia Silvae Sinicae, 42(2), 27-31. (in Chinese with English abstract) [何炎红，郭连生，田有亮 (2006).7种针阔叶树种不同 光照强度下叶绿素荧光猝灭特征. 林业科学, 42(2), 2731.]

Hong SS, Xu DQ (1999). Reversible inactivation of PSII reaction centers and the dissociation of LHCII from PSII complex in soybean leaves. Plant Science, 1147, 111-118.

$\mathrm{Hu}$ WH, Zhang SS, Xiao YA, Yan XH (2015). Physiological responses and photo-protective mechanisms of two Rhododendron plants to natural sunlight after long term shading. Chinese Journal of Plant Ecology, 39, 1093-1100. (in Chinese with English abstract) [胡文海, 张斯斯, 肖宜安, 间小红 (2015). 两种杜鹃花属植物对长期遮阴后全光 照环境的生理响应及其光保护机制. 植物生态学报, 39 , 1093-1100.]

Huang QX, Zhao S, Liu CM, Li YL (2015). Effects of shading treatments on chlorophyll fluorescence characteristics of Sabina vulgaris seedlings grown in iron tailings media. Scientia Silvae Sinicae, 51(6), 18-25. (in Chinese with English abstract) [黄秋㜀, 赵顺, 刘春梅, 李玉灵 (2015). 遮荫处理对铁尾矿基质臭柏实生苗快速叶绿素 苂光特性的影响. 林业科学, 51(6), 18-25.]

Huang SM, Wei ZF, Lu LD, Pan JT (1995). Flora Republicae Popularis Sinicae. Science Press, Beijing. 226-227. (in Chinese with English abstract) [黄淑美, 卫兆芬, 陆玲娣, 潘锦堂 (1995). 中国植物志. 科学出版社, 北京. 226-227.]

Ji LL, Lu BS, Zhou RJ, Bai ZY, Liang HY (2007). Effects of shading on photosynthetic parameters of leaf in David maple (Acer davidii Franch). Acta Horticulturae Sinica, 34(1), 173-178. (in Chinese with English abstract) [缴丽 莉, 路丙社, 周如久, 白志英, 梁海永 (2007). 遮光对 青榨槭光合速率及叶绿素荧光参数的影响. 园艺学报, 34(1), 173-178.]

Kagawa T, Sakai T, Suetsugu N, Oikawa K, Lshiguro S (2001). Arabidopsis NPL1: A phototropin homolog controlling the chloroplast high-light avoidance response. Science, 291, 
2138-2141.

Kasahara M, Kagawa T, Oikawa, Suetsugu N, Miyao M (2002). Chloroplast avoidance movement reduces photodamage in plant. Nature, 420, 829-832.

Li JP, Dong R (2011). Diurnal change of photosynthetic characteristics of three Hosta cultivars. Journal of Northeast Forestry University, 39(10), 56-58. (in Chinese with English abstract) [李金鹏，董然 (2011). 3种彩叶玉䙃光合日 变化. 东北林业大学学报, 39(10), 56-58.]

Li ZZ, Liu DH, Zhao SW, Jiang CD, Shi L (2014). Mechanisms of photoinhibition induced by high light in Hosta grown outdoors. Chinese Journal of Plant Ecology, 38, 720-728. (in Chinese with English abstract) [李志真, 刘东焕, 赵世 伟, 姜闯道, 石雷 (2014). 环境强光诱导玉䈐叶片光抑 制的机制. 植物生态学报, 38, 720-728.]

Liu JF, Yang WJ, Jing ZP, Guo QS, Jing JQ, Xue L (2011). Effects of shading on photosynthetic characteristics and chlorophyll fluorescence parameters in leaves of the endangered plant Thuja sutchuenensis. Acta Ecologica Sinica, 31, 5999-6004. (in Chinese with English abstract) [刘建锋, 杨文娟, 江泽平, 郭泉水, 金江群, 薛亮 (2011). 遮荫对濒危植物崖柏光合作用和叶绿素苂光参 数的影响. 生态学报, 31, 5999-6004.]

Murchie EH, Horton P (1998). Contrasting patterns of photo-synthetic acclimation to the light environment are dependent on the differential expression of the responses to altered irradiance and spectral quality. Plant, Cell \& Environment, 21, 139-148.

Nedbal L, Soukupova J, Kaftan D, Whitmarsh J, Trtilek M (2000). Kinetic imaging if chlorophyll fluorescence using modulated light. Photosynthesis Research, 66, 3-12.

Poorther H, Perez-Soba M (2000). The growth response of plants to elevated $\mathrm{CO}_{2}$ under non-optimal environmental conditions. Oecologia, 27, 595-607.

Richardson AD, Berlyn GP (2002). Spectral reflectance and photosynthetic properties of Betula papyrifera (Betulaceae) leaves along an elevational gradient on Mt. Mansfield. American Journal of Botany, 9, 88-94.

Wang K, Zhu JJ, Yu LZ, Sun YR, Chen GH (2009). Effects of shading on the photosynthetic characteristics and light use efficiency of Phellodendron amurense seedlings. Chinese Journal of Plant Ecology, 33, 1003-1012. (in Chinese with English abstract) [王凯, 朱教君, 于立忠, 孙一荣, 陈光 华 (2009). 遮阴对黄波罗幼苗的光合特性及光能利用
效率的影响. 植物生态学报, 33, 1003-1012.]

Wu CW, Ke TS, Chang YJ, Chang YS (2013). Chlorophyll fluorescence and leaf-air temperature difference as potential shade-tolerance indexes of ornamental plants. Journal of Computational \& Theoretical Nanoscience, 19, 30633066.

Xu DQ (2003). Several problems in the research of plant light stress. Plant Physiology Communications, 39, 493-495. (in Chinese with English abstract) [许大全 (2003). 植物 光胁迫研究中的几个问题. 植物生理学通讯, 39, 493-495.]

Xu DQ (2013). The Science of Photosynthesis. Science Press, Beijing. 94-95. (in Chinese) [许大全 (2013). 光合作用 学. 科学出版社, 北京. 94-95.]

Xu DQ, Sheng YK (1999). Light stress: Photoinhibition of photosynthesis in plants under natural conditions. In: Pessarakli M ed. Handbook of Plant and Crop Stress. Marcel Dekker, New York. 315-336.

Yang XH, Zou Q, Wang W (2001). Photoinhibition in shaded cotton leaves after exposing to high light and the time course of its restoration. Acta Botanica Sinica, 43, 1255-1259. (in Chinese with English abstract) [杨兴洪, 邹琦, 王玮 (2001). 遮荫棉花转入强光后光合作用的光 抑制及其恢复. 植物学报, 43, 1255-1259.]

Yin LQ, Hu Y, Tang GJ, Huang WC, Li SZ, Wang XQ (2010). In vitro culture and rapid propagation of Hydrangea serrata 'Preziosa'. Acta Agriculturae Shanghai, 26(1), 38-41. (in Chinese with English abstract) [殷丽青, 胡永, 汤桂 钧, 黄卫昌, 李世忠, 王新其 (2010). 优良八仙花品种 (Hydrangea serrata 'Preziosa')的离体培养与快速繁殖. 上海农业学报, 26(1), 38-41.]

Zhang CY, Fang YM, Ji HL, Ma XT (2011). Effects of shading on photosynthesis characteristics of Photinia $\times$ frasery and Aucuba japonica var. variegate. Chinese Journal of Applied Ecology, 22, 1743-1749. (in Chinese with English abstract) [张聪颖，方炎明，姬红利，马戌涛 (2011). 遮 荫处理对红叶石楠和洒金桃叶珊瑚光合特性的影响. 应用生态学报, 22, 1743-1749.]

Zhang SR (1999). A discussion on chlorophyll fluorescence kinetics parameters and their significance. Chinese Bulletin of Botany, 16, 444-448. (in Chinese with English abstract) [张守仁 (1999). 叶绿素苂光动力学参数的意义 及讨论. 植物学通报, 16, 444-448.]

责任编委: 曹 敏 责任编辑: 李 敏

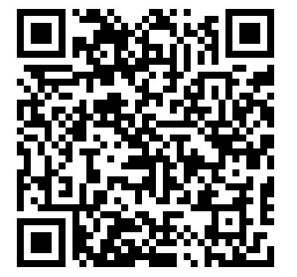

扫码向作者提问

www.plant-ecology.com 